\title{
Cell-derived microvesicles in infective endocarditis: Role in diagnosis and potential for risk stratification at hospital admission
}

\author{
Milton Henriques Guimarães Júnior ${ }^{a}$, Teresa Cristina Abreu Ferrari ${ }^{a}$, \\ Andréa Teixeira-Carvalho ${ }^{\mathrm{b}}$, Marcela de Lima Moreira ${ }^{\mathrm{b}}$, Lorena Júnia de Souza Santos ${ }^{\mathrm{b}}$, \\ Matheus Fernandes Costa-Silva ${ }^{\mathrm{b}}$, Rodrigo Matos Pinto Coelho ${ }^{\mathrm{a}}$, \\ Pedro Henrique Oliveira Murta Pinto ${ }^{a}$, Tijmen Hermen Ris ${ }^{c}$, Jonathas Teixeira Salles ${ }^{a}$, \\ Lívia Silva Araújo Passos ${ }^{a}$, Maria Carmo Pereira Nunes ${ }^{a, *}$ \\ a Programa de Pós-Graduação em Infectologia e Medicina Tropical e Departamento de Clínica Médica, Faculdade de Medicina da Universidade Federal de \\ Minas Gerais, Belo Horizonte, Brazil

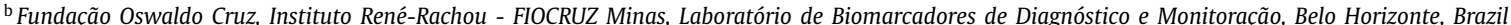 \\ ${ }^{\mathrm{c}}$ Erasmus University Rotterdam - Erasmus MC, Netherlands
}

\section{A R T I C L E I N F O}

\section{Article history:}

Accepted 10 June 2019

Available online $\mathrm{xxx}$

\section{Keywords:}

Infective endocarditis

Cell-derived microparticles;mortality

Bacterial infections

\begin{abstract}
S U M M A R Y
Objectives: To characterize the plasmatic profile of cell-derived microvesicles (MVs) at diagnosis and during the treatment of patients with infective endocarditis (IE).

Methods: Blood samples from 57 patients with IE were obtained on 3 consecutive moments: upon admission (T0), at 2 weeks (T1), and at the end of treatment (T2), and were compared with 22 patients with other bacterial infections. MPs were measured by flow cytometry and labeled for specific cell markers of CD45 (leukocytes), CD66b (neutrophils), CD14 (monocytes), CD41a (platelets), CD51 (endothelial cells), CD3 (T lymphocyte) and CD235a (erythrocytes).

Results: MVs from platelets (pltMVs), leukocytes (leukMVs), neutrophils (neutMVs), monocytes (monoMVs) and lymphocytes (lymphMVs) were significantly more elevated in the patients with IE, compared to the patients with other bacterial infections, despite comparable age, sex, blood counts and Creactive protein levels. MVs values revealed a relatively stable pattern over time in IE, except for a significant increase in leukMVs and neutMVs in T1. LeukMVs $(p=0.011)$, neutMVs $(p=0.010)$, monoMVs $(p=0.016)$ and lymphMVs $(p=0.020)$, measured at admission, were significantly higher in IE patients that died during hospitalization in comparison with those that survived. In a multivariable analyses, the levels of neutMVs remained as an independent factor associated with mortality (odds ratio 2.203; 95\% confidence interval 1.217 - 3.988; $p=0.009$ ), adjustment for heart failure during the treatment.

Conclusions: Plasma levels of pltMVs, leukMVs, neutMVs, monoMVs and lymphMVs were significantly more elevated in patients with IE than in patients with other bacterial infections at hospital admission. Furthermore, neutMVs at admission have been identified as an independent predictor of mortality in patients with IE. Thus, cell derived MPs may become an important tool in the differential diagnosis and mortality risk assessment early in the course of IE suspected cases.
\end{abstract}

(c) 2019 The British Infection Association. Published by Elsevier Ltd. All rights reserved.

\section{Introduction}

Infective endocarditis (IE) is the microorganism infection of the endocardium known for its high morbidity and mortality in spite of treatment. ${ }^{1}$ The clinical presentation and outcome are largely determined by the immune and inflammatory response influenced by the host-pathogen interaction. The complex pathogenesis of

\footnotetext{
* Corresponding author.

E-mail address: mcarmo@waymail.com.br (M.C.P. Nunes).
}

this condition is a challenge, and understanding the immuneinflammatory cell pathways can be a step closer to improve disease management.

In the last decades, studies have demonstrated a role of cellderived microvesicles (MVs) in the immune-inflammatory response in several diseases. MVs are small vesicles, typically around 100$1000 \mathrm{~nm}$ in size, released from the plasma membrane by activated, injured, or apoptotic cells. ${ }^{2,3}$ Although there is no consensual definition of $\mathrm{MVs}^{4}{ }^{4} \mathrm{MVs}$ are larger than exosomes, their density is unknown and they are usually isolated at 10,000 to $20,000 \mathrm{x}$ g by 
centrifugation. MVs are often called microparticles (MPs), although the term "microparticles" has also been used for total populations of vesicles isolated from human plasma at $100,000 \times \mathrm{g}$ and such populations will contain exosomes. ${ }^{5}$

The release of MVs have been demonstrated in physiological ${ }^{6}$ and pathological conditions acting as intercellular messengers. ${ }^{7}$ The number and constitution of the released MVs depends on the cell type and its state, and on the environmental conditions. ${ }^{8}$ MVs have been studied in sepsis, ${ }^{9-12}$ where they were demonstrated to be associated with microvascular dysfunction, organ damage ${ }^{9}$ and coagulation abnormalities. ${ }^{13}$ MVs have also been associated with thromboembolic events in patients with cancer, ${ }^{14}$ and with plaque instability in coronary artery disease. ${ }^{15}$ Some studies demonstrated that the total number of MVs, including the MVs derived from platelets, monocytes and endothelium, was significantly higher in patients with type 2 diabetes mellitus than in non-diabetic controls. $^{16,17}$ In chronic obstructive lung disease, endothelium derived MVs have been associated with the grade of lung destruction, airflow limitation and disease exacerbation. ${ }^{18}$

Taking all together, it is possible that MVs participate in the pathophysiology of IE. However, up to this date, there are no studies evaluating the profile of plasmatic MVs in IE. Therefore, the aim of our study was to characterize the profile of MVs released by different cells in IE, compare their kinetics during the disease treatment, and evaluate their potential for predicting clinical outcome.

\section{Methods}

Between August 2011 and January 2017, 65 patients with definite IE, according to the Dukeś Modified Criteria, ${ }^{19}$ consecutively admitted to the University Hospital, Federal University of Minas Gerais, Brazil were evaluated for inclusion in the study. Exclusion criteria were IE patients who were taking antibiotics for more than one week prior to the moment of inclusion in the study, and those who died or underwent cardiac surgery before the collection of the first blood sample for MVs measurement. The Institutional Ethics Committee approved the study protocol and written informed consent was obtained from all the participants.

After being included in the study, the patients were followed during hospitalization, and their clinical, laboratorial and echocardiographic data were recorded in the study protocol. Blood samples for MVs measurement were obtained at 3 consecutive times, as follows:

\footnotetext{
T0 At the diagnosis of infective endocarditis At the 15th day of antibiotic treatment Before hospital discharge
}

Blood sample for MVs quantification was collected before the beginning of the antibiotics or within the first week of treatment, we meant that the T0 sample was collected at the time of diagnosis, preferably before the beginning of the antibiotics. Patients who had already been taking antibiotics at the time of diagnosis had the T0 sample collected only if they had been taking it for less than 7 days, otherwise they were excluded from the study. At the later situation, it was not collected at the same day for every single patient because the time from antibiotic initiation and the definition of the IE diagnosis varied among them. Then, T1 samples were collected at day 15th of antibiotic treatment for every single patient, and T2 after completing the treatment, before hospital discharge.

The endpoint analyzed in this study was overall mortality related to any complication of IE during hospitalization.

The data of the patients with IE were compared with those of a control group, consisting of 22 patients with other bacterial infections that presented with fever and elevated C-reactive protein (CRP) serum levels. The other infections comprised pyelonephritis
(8 cases), pneumonia (7 cases), catheter-related bloodstream infection (6 cases), and monoarthritis (1 case) without any organ dysfunction and any other sign of severity. Samples from the control group were collected only once, i.e. by the time of their enrollment in the study. As for the IE group, blood sample for MVs quantification was collected before the beginning of the antibiotics or within the first week of the use of these agents.

\section{Sample preparation and MVs measurement}

Citrated peripheral blood samples $(3.2 \mathrm{~mL})$ was centrifuged at $3000 \times \mathrm{g}$ for $15 \mathrm{~min}$, and then the plasma was cooled at $-20^{\circ} \mathrm{C}$ before storage at $-80^{\circ} \mathrm{C}$. Immediately before analyses, the samples were thawed at $37^{\circ} \mathrm{C}$ and were further centrifuged at $13,000 \times \mathrm{g}$ for $3 \mathrm{~min}$ to obtain platelet-free plasma. The latter was diluted $1: 3$ in citrated phosphate buffered saline (PBS) containing heparin and centrifuged at $14,000 \times \mathrm{g}$ for $90 \mathrm{~min}$ at $15^{\circ} \mathrm{C}$. The resultant MVs pellet was then resuspended in $1 \times$ annexin $\mathrm{V}$ binding buffer (BD Biosciences, CA).

MVs were measured by flow cytometry. MVs selection was based on particle size, presence of a common surface marker (phosphatidylserine) and specific surface antigens according to the cell origin as described elsewhere. ${ }^{20-22}$ In the first step, the MVs isolated from plasma were gated (R1) based on their forward (FSC) and side (SSC) scatter distribution compared to the distribution of synthetic 0.7-0.9 $\mu \mathrm{m}$ SPHERO $^{\mathrm{TM}}$ Amino Fluorescent Particles (Spherotech Inc. Libertyville, IL). After that, events present in $\mathrm{R} 1$ were accessed for their positive staining for annexin V (BD Bioscience, CA), which binds to phosphatidylserine. To properly place gates, mouse IgG PE conjugated isotype control monoclonal antibodies (mAbs) were used. Finally, annexin $V+$ events were gated with conjugated mAbs against the cell markers CD45-PE (leukocytes), CD66b-PE (neutrophil), CD14-PercCP (monocytes), CD3-PE (T lymphocyte) CD41a-PercCP (platelets), CD235a-PECy5 (erythrocytes) and CD51/61-PE (endothelial cells).

All reagents and $m A b s$ used in the flow cytometry experiments were from BD Biosciences (Becton-Dickinson, CA), unless otherwise stated. The samples were analysed in a FACScalibur flow cytometer (Becton-Dickinson, CA). Over 100,000 events were acquired on each sample to reach at least 2000 events within the MVs gate.

The cytometer was set to operate at a high flow rate setting for $60 \mathrm{~s}$ for each sample. The number of MVs/ $\mu \mathrm{L}$ of plasma was calculated as described elsewhere ${ }^{23}: \mathrm{MVs} / \mu \mathrm{L}=(N \times 400) /(60 \times 100)$, in which $\mathrm{N}$ is the number of events, 400 is the total volume of the tube before analysis, 60 is the sample volume analyzed, and 100 is the original volume of MPs suspension used to perform the phenotyping protocol.

\section{Normal reference values}

As normal reference values we used the ones obtained in the same research laboratory (Instituto René Rachou, FIOCRUZ Minas), with similar protocol analyses, derived from 30 healthy blood donors. MVs reference values were: until 75 counts $/ \mu \mathrm{L}$ for leukocytes MVs (leukMVs); until 75 counts/ $\mu \mathrm{L}$ for neutrophils MVs (neutMVs); until 75 counts/ $\mu \mathrm{L}$ for monocytes MVs (monoMVs); until 45 counts/ $\mu \mathrm{L}$ for T lymphocytes MVs (lymphMVs); until 90

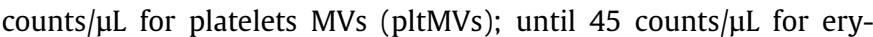
throcytes MVs (eryMVs); and until 54 counts $/ \mu \mathrm{L}$ for endothelium MVs (endoMVs).

\section{Statistical analysis}

Categorical variables, expressed as numbers and percentages, were compared using chi-square testing, whereas continuous data, expressed as median and interquartile range (IQR), were compared 
Table 1

Baseline characteristics of the study population.

\begin{tabular}{|c|c|}
\hline Variable & Value \\
\hline Age (years) & $50(39-64)$ \\
\hline Male & $33(58.0)$ \\
\hline Diabetes mellitus & $9(15.8)$ \\
\hline Chronic renal disease & $13(22.8)$ \\
\hline \multicolumn{2}{|l|}{ Predisposing conditions } \\
\hline Rheumatic valve disease & $17(29.8)$ \\
\hline Degenerative valve disease & $9(15.7)$ \\
\hline Mitral valve prolapse & $11(19.2)$ \\
\hline Congenital heart disease & $5(8.7)$ \\
\hline Central venous catheter & $7(12.0)$ \\
\hline Previous infective endocarditis & $2(3.4)$ \\
\hline \multicolumn{2}{|l|}{ Clinical findings } \\
\hline Fever & $45(80.0)$ \\
\hline Weightloss & $30(53.0)$ \\
\hline Anorexia & $37(65.0)$ \\
\hline Musculoskeletal manifestation & $12(21.0)$ \\
\hline Heart murmur & $44(77.0)$ \\
\hline \multicolumn{2}{|l|}{ Microbiologic etiology } \\
\hline Coagulase-negative Staphylococcus & $15(26.3)$ \\
\hline Streptococcusspp & $7(12.3)$ \\
\hline Staphylococcus aureus & $6(10.5)$ \\
\hline Gram negative rods & $5(8.8)$ \\
\hline Enterococcus spp & $3(5.3)$ \\
\hline Fungi & $2(2.5)$ \\
\hline Negative culturefindings & $17(29.8)$ \\
\hline \multicolumn{2}{|l|}{ Complications and outcomes } \\
\hline In-hospital death & $17(30.0)$ \\
\hline Early surgery & $25(44.0)$ \\
\hline Development of heart failure & $29(51.0)$ \\
\hline Fever $>10$ days (on treatment) & $8(14.0)$ \\
\hline Neurologic event & $11(19.0)$ \\
\hline
\end{tabular}

Data are expressed as absolute numbers (percentage) and median (interquartile range).

using the Mann-Whitney U test, as appropriate. Logistic regression model was constructed to test the independent association between MVs concentrations and death, after adjustment for other covariates. The data were analyzed using SPSS 23.0 statistical software (SPSS, Chicago, IL) and Prism GraphPad software, version 5 (San Diego, CA).

\section{Results}

The baseline characteristics of the 57 patients classified as having definitive IE are shown in Table 1. Median age of the IE patients was 50 years (IQR 39-64 years), and 33 (58\%) individuals were male. Native valve IE was observed in 32 (56\%) cases, followed by prosthetic valve IE in 15 patients (26\%) and devicerelated IE in 10 (17.5\%). The most common predisposing condition was rheumatic valve disease $(29.8 \%)$ and the most common comorbidity was chronic renal disease (22.8\%). One patient had HIV infection, none was illicit drug user. The most prevalent agent was coagulase-negative Staphylococcus, which was isolated in 15 (26\%) of the cases, followed by Streptococcus spp (12\%) and S. aureus (10\%). Culture-negative IE represented $29.8 \%$ of the cases.

Transesophageal echocardiography was performed in 47 (82\%) of the patients and transthoracic echocardiogram in the remaining 10 (17.5\%). Vegetations were identified in 49 patients (86\%) and 20 (35\%) vegetations were described as $>10 \mathrm{~mm}$.

There was no significant difference between the IE group and the group with other bacterial infections regarding age, CRP levels, hemoglobin concentrations, white blood cell counts, platelets counts and creatinin levels (Table 2).

A high proportion $(>84 \%)$ of patients in the IE group and in the control group of the other bacterial infections presented levels of all MVs phenotypes above the reference values. The comparison
Table 2

Demographical and laboratorial features of the patients with IE compared with the patients with other bacterial infections.

\begin{tabular}{|c|c|c|c|}
\hline Variable & Cases $(n=57)$ & Controls $(n=22)$ & $P$ value \\
\hline Age (years) & $51.0(39-64)$ & $56.6(23-67)$ & 0.411 \\
\hline Male (n/\%) & $33(58)$ & $10(41)$ & 0.457 \\
\hline C-reactive protein $(\mathrm{mg} / \mathrm{L})$ & $76(37-183)$ & $139(50-240)$ & 0.610 \\
\hline Hemoglobin (g/dl, mean) & $10(9-11)$ & $11.5(9-13)$ & 0.007 \\
\hline Leukocytosis $\left(\mathrm{x} 10^{3} / \mu \mathrm{l}\right)$ & $11(7-14)$ & $10(5-13)$ & 0.218 \\
\hline Platelets $\left(\times 10^{3} / \mu \mathrm{l}\right)$ & $181(137-251)$ & $186(115-267)$ & 0.732 \\
\hline Creatinin (mg/dl) & $1.08(0.8 / 1.7)$ & $0.97(0.8 / 1.2)$ & 1.000 \\
\hline \multicolumn{4}{|l|}{ Microvesicles (counts $/ \mu \mathrm{L}$ ) } \\
\hline Leukocytes & $560(239-832)$ & $313(200-503)$ & 0.032 \\
\hline Neutrophils & $275(138-478)$ & $135(94-265)$ & 0.023 \\
\hline Monocytes & $202(121-428)$ & $110(82-210)$ & 0.051 \\
\hline Lymphocytes & $200(130-303)$ & $119(58-241)$ & 0.034 \\
\hline Erythrocytes & $166(85-305)$ & $111(58-217)$ & 0.110 \\
\hline Endothelium & $428(244-728)$ & $242(212-380)$ & 0.054 \\
\hline Platelets & $536(341-1119)$ & $317(218-492)$ & 0.020 \\
\hline
\end{tabular}

Data are expressed as the median (interquartile range).

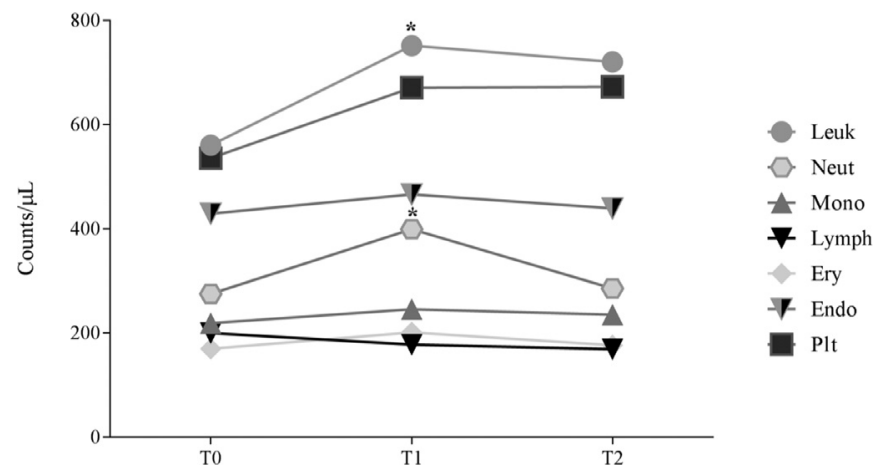

Fig. 1. MVs levels from different phenotypes, at T0 (admission), T1 (two weeks of treatment) and T2 (end of treatment) in IE patients. Data are expressed as median. Leuk = microvesicles from leukocytes; Neut $=$ microvesicles from neutrophils; Mono $=$ microvesicles from monocytes; $L y m p h=$ microvesicles from $\mathrm{T}$ lymphocytes; Ery = microvesicles from erytrocites; Endo = microvesicles from endothelium; $\mathrm{Plt}=$ microvesicles from platelets. Statistically significant differences $(p<0.05)$ between the times T0 and T1 are indicated by an asterisk above the symbol related to each group.

of the MV counts between the group of other bacterial infections and IE patients (at admission) demonstrated significantly higher levels of the leukMVs $(p=0.032)$, neutMVs $(p=0.023)$, lymphMVs $(p=0.034)$ and pltMVs $(p=0.020)$ in the IE patients

\section{Microvesicle counts during treatment}

During hospital stay, 17 patients died with an overall mortality rate of $30 \%$. Twenty-five patients (44\%) were submitted to cardiac surgery, chiefly due to development of heart failure (51\%). The median time between the diagnosis and the surgery was 9 days (IQR 3-17 days). Neurologic events occurred in 11 (19.3\%) patients and the most prevalent was ischemic stroke, which was observed in 9 (15.8\%) individuals.

Fig. 1 shows MVs levels during treatment course. LeukMVs, pltMVs and endoMVs presented the highest levels. There were a higher count of leukMVs $(p=0.049)$ and neutMVs $(p=0.033)$ in T0 compared with $\mathrm{T} 1$.

To analyze the potential value of MVs in predicting early mortality, initially we compared MV levels at admission (T0) and at $\mathrm{T} 1$ between the patients who died within the first 2 weeks and those who survived at least 2 weeks after diagnosis. We found that the counts of leukMVs $(p=0.018)$, neutMVs $(p=0.009)$, monoMVs $(p=0.015)$ and lymphMVs $(p=0.019)$ measured at T0 were significantly higher in the patients who died compared to those who 


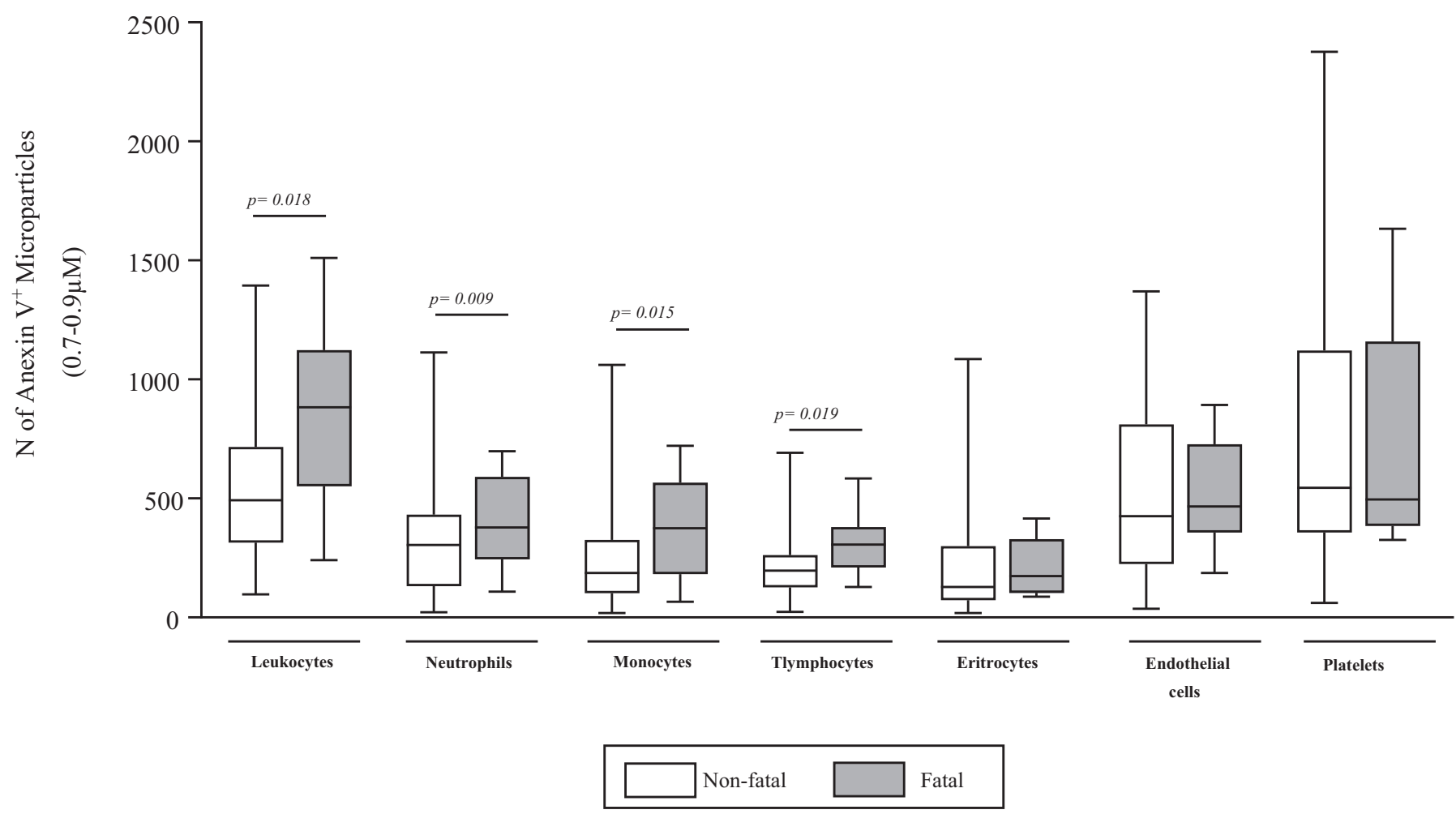

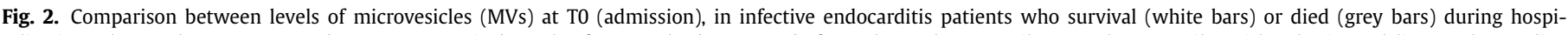

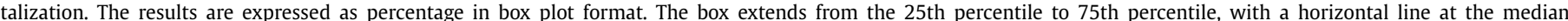

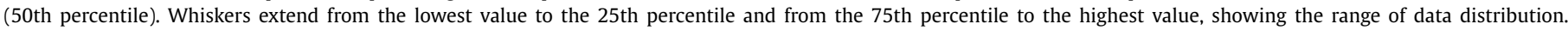
Statistical significance is indicated in each graph.

survived (Fig. 2). MV levels at different times (T0, T1, T2) are shown in Fig. 3 (A and B). Although pltMVs levels at $\mathrm{T} 1$ were higher in those who died, this difference was not significant, as there were a wide variation in pltMVs during the disease evolution. Additionally, mean platelet volume (MPV) values, which were $8.7 \pm 2.6 \mathrm{fL}$ presented no differences between the patients who died and survived.

There were no significant differences in MV levels at admission concerning the need of surgery, cerebral event, or development of heart failure. In the logistic regression analyses, the levels of neutMVs at admission remained as an independent predictor of death, after adjustment for worsening of heart failure during the course of IE (odds ratio 2.203; 95\% CI 1.217-3.988; $p=0.009$ ).

\section{Discussion}

As far as we know, this is the first study describing the plasma concentrations and kinetics of MVs in patients with IE, and also comparing IE MV levels with those observed in other bacterial infections. All MVs phenotypes demonstrated elevated plasma levels during infection (IE and the other bacterial infections groups) when compared with reference values defined in a healthy population. Despite comparable age, sex, white blood cell count and CRP levels, we observed significantly higher baseline levels of leukMVs, neutMVs, lymphMVs and pltMVs in the IE group. This suggests a greater activation of these cells in IE resulting in higher MVs production. MVs levels stratified by mortality showed higher levels of leukMVs, neutMVs, monoMVs and lymphMVs at admission in the patients who died compared with those who survived. After adjustment for development of heart failure, which is the main complication of IE, neutMVs at baseline remained predictor of death.

Extracellular vesicles (EVs) encompass a broad range of vesicles released from cells. ${ }^{3}$ EVs can be classified into different subsets according to their size, cellular origin, content or the mechanism leading to their formation. At present, at least 3 main subgroups of EVs have been defined. MVs, also referred to as microparticles, are vesicles typically around $100-1000 \mathrm{~nm}$ in size.,24,25 Exosomes are smaller cell-derived vesicles that are present in all biological fluids. Their diameter is between 30 and $100 \mathrm{~nm}$, the density ranges between 1.13 and $1.19 \mathrm{~g} / \mathrm{ml}$ and the morphology has been described as cup-shaped. Apoptotic bodies are larger vesicles containing nuclear materials. In our study we have analyzed EVs from $500 \mathrm{~nm}$ to $900 \mathrm{~nm}$, thus our population was constituted of MVs, without overlap with exosomes.

In the IE patients, the highest levels of MVs were derived from leukocytes, followed by those derived from platelets and endothelial cells. Studies that evaluated patients with sepsis ${ }^{26}$ and meningococcal disease ${ }^{27}$ demonstrated similar findings. The elevation of neutMVs levels found in our patients with IE is also similar to the results of Timár et al. ${ }^{28}$ that demonstrated a 6 -fold increase in the release of neutMVs, comparing patients with $S$. aureus bacteremia with healthy individuals.

In some studies concerning other diseases in which MVs counts were performed, the authors observed a predominant increase in pltMVs differently from what was observed in the present study, in which leukMV counts were very similar to those of pltMVs in both cases and controls with other infections. As those studies included patients who were critically-ill, including meningococcal infection with septic shock, ${ }^{27}$ acute Plasmodium vivax malaria, ${ }^{20}$ and sepsis admitted to an intensive care unit, ${ }^{26}$ and probably presented an acute phase response more exacerbated than that exhibited by our cases, the intensity of the acute phase response and the more acute character of the infection may be possible explanations for the observed difference. However, the possibility of influence by pre-analytical factors cannot be ruled out. 
A

Non-fatal

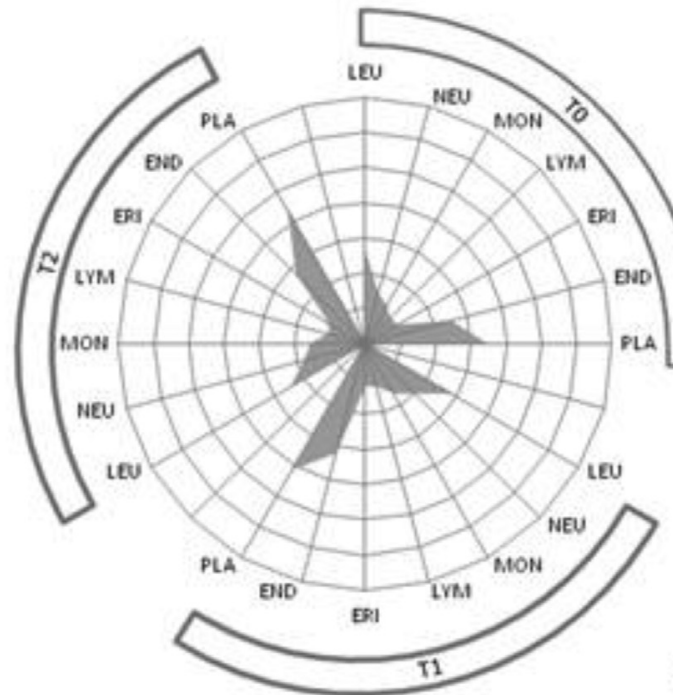

Fatal

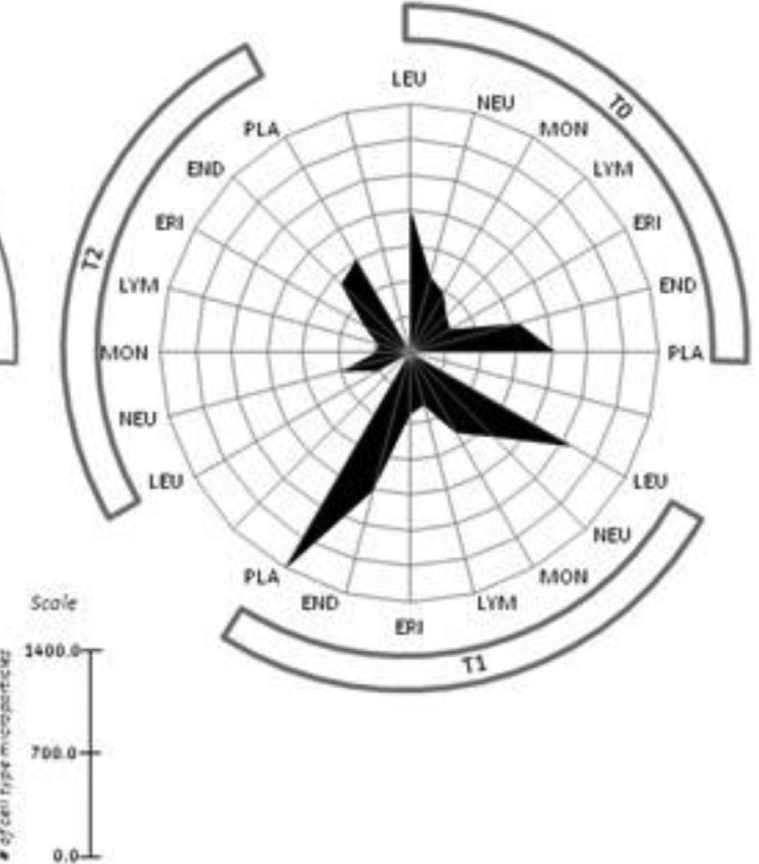

B

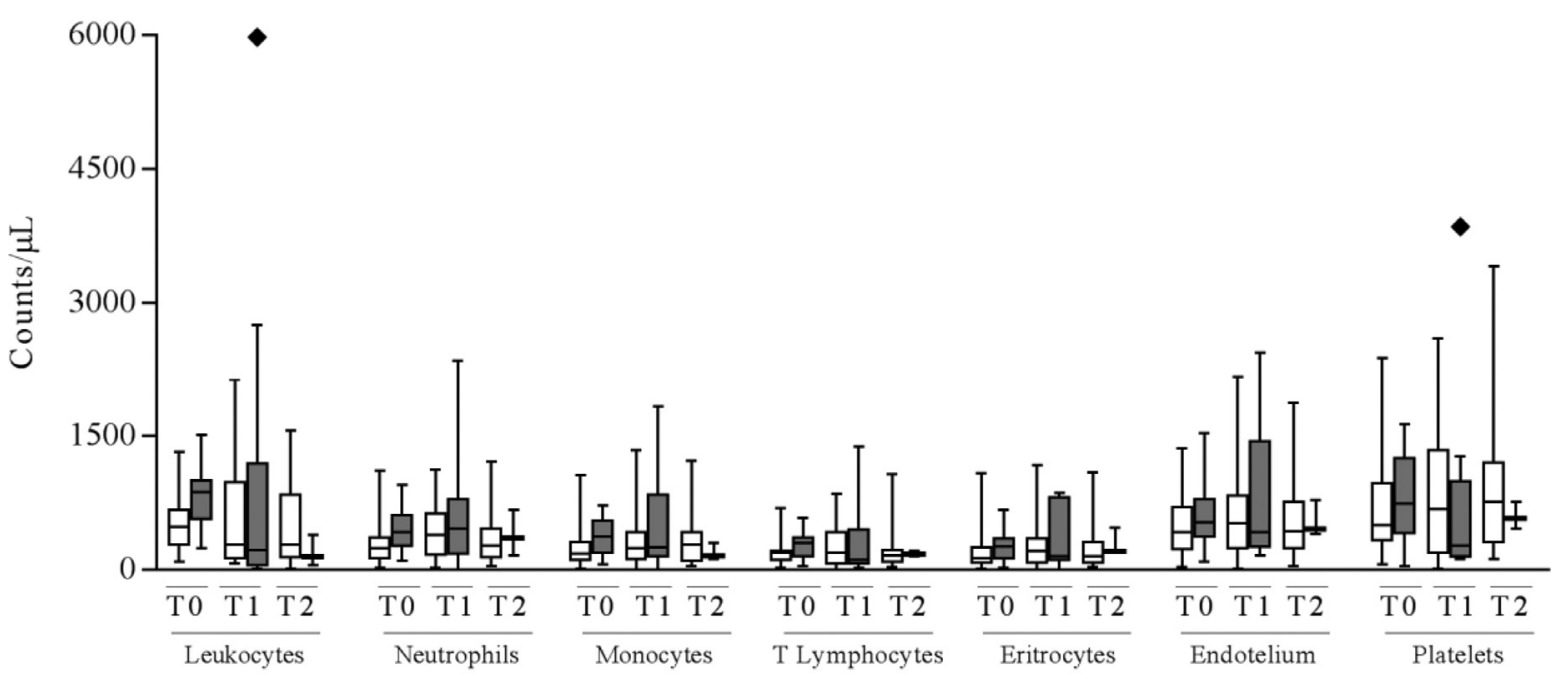

Non-fatal $\square$ Fatal

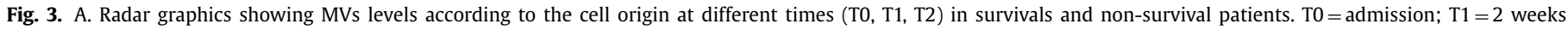

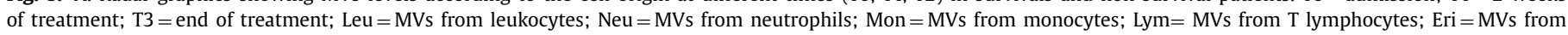
erytrocites; End $=$ MVs from endothelium; Pla $=$ MVs from platelets.

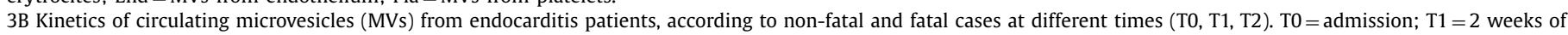

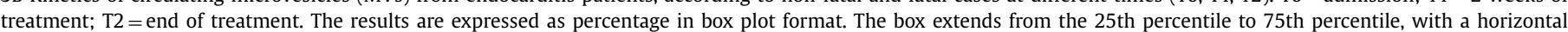

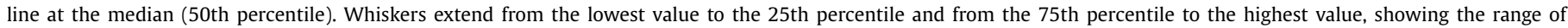
data distribution. Outliers values was plotted as individual points.

\section{Microvesicles kinetics during the treatment}

There are few studies reporting MVs kinetics, determined by flow cytometry, during treatment of infectious diseases; furthermore, most of them are cross-sectional and none of them in IE. We observed increase in the leukMVs and neutMVs serum concentrations from admission (T0) to the end of the second week of treatment in the IE patients (T1). An interesting observation was the course of pltMVs in surviving patients. Overtime, the pltMVs tended to increase which may represent a protective role of pltMVs during IE. This in agreement with a study that found a negative correlation between pltMVs and organ dysfunction in the 
patients who died due to severe sepsis. ${ }^{29}$ However, on T1, nonsurviving group had an important increase in pltMVs as well, but the pltMV levels were lowered again at T2.

It can be speculated a possible influence of cardiac surgery on this finding as in our cohort, $44 \%$ of the patients underwent surgery, in a median of 9 days after the diagnosis (before obtaining data of T1). However, the half-life of MVs seems to be very short. Rank et al. ${ }^{30}$ reported that pltMVs half-life in humans is $5.8 \mathrm{~h}^{30}$ A shorter time (90\% clearance after $30 \mathrm{~min}$ ) was reported in an animal study of exosomos released by erythrocytes. ${ }^{31}$ Furthermore, Fu et al. ${ }^{32}$ did not find any difference in the total MVs concentrations comparing the MP levels before surgery with those obtained 12 and $72 \mathrm{~h}$ after the procedure. A possible explanation for this finding is the above described short half-life of MVs. Thus, we cannot attribute the enhancement of leukMV and neutMV levels observed in our study to the surgical procedure. Nevertheless, it is known that cardiopulmonary bypass surgery provokes an inflammatory state that is able to induce MVs release. ${ }^{33}$

\section{Value of microparticles in predicting mortality}

The mechanisms that stimulate MVs release are cell activation and apoptosis, in a process involving cytokine and endotoxin release, complement lysis, oxidative stress and high shear stress, ${ }^{34,35}$ which may predict disease severity.

The association between MVs levels and clinical outcomes has been investigated in other diseases. Zafrani et al. ${ }^{36}$ demonstrated that preventing the release of MVs was associated with a decrease in microvasculature dysfunction and higher survival in animal models of sepsis. Delabranche et al. ${ }^{13}$ found increased leukMVs levels in patients with sepsis that developed disseminated intravascular coagulation. In agreement with our finding that neutMVs were predictors of mortality during hospitalization, a previous study showed a high mortality rate in septic mice injected with neutMVs. ${ }^{37}$

Other authors have explored the mechanisms that could explain the association between leukMVs, neutMVs, monoMVs or lymphMvs and high mortality. When looking for mechanisms, is important to take into account that the role of MVs depends on their composition, which is related to the cell of origin and the target cell. MVs from $\mathrm{T}$ lymphocytes are capable of inducing pro-inflammatory peptides, nitric oxide-synthesis and cyclooxygenase- 2 expressions, especially in the middle layer of the vessels. ${ }^{38}$ MonoMVs induce, in a concentration-dependent manner, reactive oxygen species production, cytokine release and nuclear factor kappa B (NF-Kb) activation in monocytes and macrophages. ${ }^{39}$

Illustrating the complexity of MVs effects, neutMVs have been reported to be pro or antiinflamatory, depending on the target cell. ${ }^{40}$ Gasser et $a l .{ }^{41}$ observed that neutMVs are capable of provoking a dose-dependent release of the anti-inflammatory cytokine transforming growth factor $\beta 1$ (TGF- $\beta 1$ ) on macrophages, as well as a decrease in the release of interleukin (IL)-6, CXCL-8 and tumor necrosis factor alpha (TNF- $\alpha$ ). Johnson et al. ${ }^{37}$ demonstrated in animal model that the administration of neutMVs was associated with increase in bacterial load and IL-10 levels, and a decrease in macrophage activation. Otherwise, it was demonstrated that neutMVs caused a pro-inflammatory response on platelets ${ }^{42}$ and endothelial cells. ${ }^{43}$ As an effect of the IE antimicrobial therapy, the ongoing bacteria destruction and clearance cause continuing antigen exposure, increasing the stress and pro-inflammatory factors release, resulting in activation of neutrophils and elevation of neutMV levels during the course of treatment, especially during the initial phase when the destruction of bacteria is more intense. However, further research, comparing the MVs levels with the release of cytokines, endotoxins, complement lysis, oxidative stress and high shear stress could clarify their effects. Irrespective of their role, plasmatic counts of MVs, specifically neutMVs, may be a useful prognostic tool for risk assessment during the hospitalization of IE patients. The possible mechanisms implicated in this poor outcome, as well as the final MVs effect (by enhancing a pro or an antiinflamatory state) deservers more investigation.

In this context, knowledge about the role of platelets in the development and severity of various conditions, in addition to thrombosis, continues to appear, especially in relation to the areas of inflammation and immune response. ${ }^{44}$ As elevated mean platelet volume (MPV) indicates increased platelet activation, this index has been studied in several disorders. Some authors observed that the MPV was higher in patients with IE than in health controls and decreased significantly after treatment. ${ }^{45,46}$ It has also been demonstrated that IE patients who present embolic events had increased MPV values, compared to those who do not present this complication. ${ }^{47,48}$ Furthermore, previous history of IE, S. aureus infection, end-stage renal disease, depressed left ventricular ejection fraction, early surgical intervention, vegetation size $\geq 10 \mathrm{~mm}$, presence of perivalvular abscess, higher onadmission platelet count, CRP and MPV levels emerged as independent predictors of in-hospital poor outcomes. ${ }^{48}$ Thus, high MPV values may be a biomarker of unfavorable outcome in IE, especially regarding the increased risk of thromboembolic events. Additionally, it is possible to speculate that platelets may play a role in the mortality of these patients. However, it was demonstrated that several diseases, including local or systemic infections and many drugs may potentially affect MPV levels. ${ }^{48}$ Thus, further studies taking into account the factors that may affect MPV are necessary to assess the actual associations between MPV and IE.

The lower limit of detection of the cytometer that was used in our investigation $(0.7 \mu \mathrm{m})$ did not allow the detection of smaller MVs, which is a limitation of the present study. In addition, our sample size and number of deaths limited the possibility to adjust for more variables in the multivariable analyses.

\section{Conclusions}

This study demonstrated that MVs derived from platelets, leukocytes, neutrophils, monocytes and lymphocytes were significantly more elevated in patients with IE than in patients with other bacterial infections. MVs concentration from leukocytes and neutrophils were higher at IE diagnosis than at 2 weeks of treatment. Moreover, MVs from neutrophils have been identified as an independent predictor of mortality in patients with IE. Therefore, cell derived MVs could become an important tool in the differential diagnosis and mortality risk assessment of patients with suspected IE at admission. However, studies with larger sample size are needed before drawing definitive conclusions.

\section{References}

1. Abdulhak AAB, Baddour LM, Erwin PJ, Hoen B, Chu VH, Mensah GA, et al. Global and regional burden of infective endocarditis, 1990-2010: a systematic review of the literature. Glob Heart 2014;9(1):131-43.

2. Mooberry MJ, Key NS. Microparticle analysis in disorders of hemostasis and thrombosis. Cytometry Part A 2016;89(2):111-22.

3. Van der Pol E, Böing A, Gool E, Nieuwland R. Recent developments in the nomenclature, presence, isolation, detection and clinical impact of extracellular vesicles. J Thromb Haemost 2016;14(1):48-56.

4. Gould SJ, Raposo G. As we wait: coping with an imperfect nomenclature for extracellular vesicles. J Extracell Vesicles 2013;2.

5. Van der Pol E, Böing AN, Harrison P, Sturk A, Nieuwland R. Classification, functions, and clinical relevance of extracellular vesicles. Pharmacol Rev 2012;64(3):676-705.

6. Berckmans RJ, Nieuwland R, Boing A, Romijn F, Hack CE, Sturk A Cell-derived microparticles circulate in healthy humans and support low grade thrombin generation. Thrombosis Haemostasis-Stuttgart- 2001;85(4):639-46.

7. Liu ML, Williams KJ, Werth VP. Microvesicles in autoimmune diseases. Adv Clin Chem 2016;77:125-75. 
8. Ratajczak J, Miekus K, Kucia M, Zhang J, Reca R, Dvorak P, et al. Embryonic stem cell-derived microvesicles reprogram hematopoietic progenitors: evidence for horizontal transfer of mRNA and protein delivery. Leukemia 2006;20(5):847-56.

9. Souza ACP, Yuen PS, Star RA. Microparticles: markers and mediators of sepsis-induced microvascular dysfunction, immunosuppression, and AKI. Kidney Int 2015;87(6):1100.

10. Reid VL, Webster NR. Role of microparticles in sepsis. Br J Anaesth 2012;109(4):503-13.

11. Zafrani L, Ince C, Yuen PST. Microparticles during sepsis: target, canary or cure? Intensive Care Med 2013;39(10):1854-6.

12. Mostefai HA, Meziani F, Mastronardi ML, Agouni A, Heymes C, Sargentini C, et al. Circulating microparticles from patients with septic shock exert protective role in vascular function. Am J Respir Crit Care Med 2008;178(11):1148-55.

13. Delabranche X, Boisramé-Helms J, Asfar P, Berger A, Mootien Y, Lavigne T, et al. Microparticles are new biomarkers of septic shock-induced disseminated intravascular coagulopathy. Intensive Care Med 2013;39(10):1695-703.

14. Zwicker JI, Liebman HA, Neuberg D, Lacroix R, Bauer KA, Furie BC, et al. Tumor-derived tissue factor-bearing microparticles are associated with venous thromboembolic events in malignancy. Clin Cancer Res 2009;15(22):6830-40.

15. Mallat Z, Benamer H, Hugel B, Benessiano J, Steg PG, Freyssinet J-M, et al. Elevated levels of shed membrane microparticles with procoagulant potential in the peripheral circulating blood of patients with acute coronary syndromes. Circulation 2000;101(8):841-3.

16. Deng F, Wang S, Zhang L. Endothelial microparticles act as novel diagnostic and therapeutic biomarkers of diabetes and its Complications: a literature review. Biomed Res Int 2016;2016 Article ID 9802026.

17. Li S, Wei J, Zhang C, Li X, Meng W, Mo X, et al. Cell-Derived microparticles in patients with type 2 diabetes mellitus: a systematic review and meta-analysis. Cell Physiol Biochem 2016;39(6):2439-50.

18. Takahashi T, Kubo H. The role of microparticles in chronic obstructive pulmonary disease. Int J Chron Obstruct Pulmon Dis 2014:9:303-14.

19. Li JS, Sexton DJ, Mick N, Nettles R, Fowler VG, Ryan T, et al. Proposed modifications to the duke criteria for the diagnosis of infective endocarditis. Clin Infect Dis 2000;30(4):633-8.

20. Campos FM, Franklin BS, Teixeira-Carvalho A, Agnaldo Filho L, de Paula SC, Fontes CJ, et al. Augmented plasma microparticles during acute plasmodium vivax infection. Malar J 2010;9(1):327.

21. Combes V, Taylor TE, Juhan-Vague I, Mège I-L, Mwenechanya J, Tembo M, et al. Circulating endothelial microparticles in malawian children with severe falciparum malaria complicated with coma. JAMA 2004;291(21):2542-4

22. Couper KN, Barnes T, Hafalla JC, Combes V, Ryffel B, Secher T, et al. Parasite-derived plasma microparticles contribute significantly to malaria infection-induced inflammation through potent macrophage stimulation. PLoS Pathog 2010;6(1):e1000744.

23. Campos FM, Franklin BS, Teixeira-Carvalho A, Filho AL, de Paula SC, Fontes CJ, et al. Augmented plasma microparticles during acute plasmodium vivax infection. Malar J 2010;9:327.

24. Yáñez-Mó M, Siljander PR-M, Andreu Z, Bedina Zavec A, Borràs FE, Buzas EI, et al. Biological properties of extracellular vesicles and their physiological functions. J Extracell Vesicles 2015;4(1):27066.

25. Zaldivia MT, McFadyen JD, Lim B, Wang X, Peter K. Platelet-derived microvesicles in cardiovascular diseases. Front Cardiovasc Med 2017:4:74.

26. Zhang Y, Meng H, Ma R, He Z, Wu X, Cao M, et al. Circulating microparticles, blood cells, and endothelium induce procoagulant activity in sepsis through phosphatidylserine exposure. Shock 2016;45(3):299-307.

27. Nieuwland R, Berckmans RJ, McGregor S, Böing AN, Romijn FPTM, Westendorp RG, et al. Cellular origin and procoagulant properties of microparticles in meningococcal sepsis. Blood 2000;95(3):930-5.

28. Timár CI, Lörincz ÁM, Csépányi-Kömi R, Vályi-Nagy A, Nagy G, Buzás EI, et al. Antibacterial effect of microvesicles released from human neutrophilic granulocytes. Blood 2013;121(3):510-18.
29. Soriano AO, Jy W, Chirinos JA, Valdivia MA, Velasquez HS, Jimenez JJ, et al. Levels of endothelial and platelet microparticles and their interactions with leukocytes negatively correlate with organ dysfunction and predict mortality in severe sepsis. Crit Care Med 2005;33(11):2540-6.

30. Rank A, Nieuwland R, Crispin A, Grützner S, Iberer M, Toth B, et al. Clearance of platelet microparticles in vivo. Platelets 2011;22(2):111-16.

31. Willekens FL, Werre JM, Kruijt JK, Roerdinkholder-Stoelwinder B, Groenen-Döpp YA, van den Bos AG, et al. Liver kupffer cells rapidly remove red blood cell-derived vesicles from the circulation by scavenger receptors. Blood 2005; 105(5):2141-5.

32. Fu L, Hu X-X, Lin Z-B, Chang F-J, Ou Z-J, Wang Z-P, et al. Circulating microparticles from patients with valvular heart disease and cardiac surgery inhibit endothelium-dependent vasodilation. J Thorac Cardiovasc Surg 2015;150(3):666-72.

33. Bronicki RA, Hall M. Cardiopulmonary bypass-induced inflammatory Response: pathophysiology and treatment. Pediatr Crit Care Med 2016;17(8_suppl):S272-S2S8.

34. Horstman LL, Jy W, Jimenez JJ, Bidot C, Ahn YS. New horizons in the analysis of circulating cell-derived microparticles. Keio J Med 2004;53(4):210-30.

35. Miyazaki Y, Nomura S, Miyake T, Kagawa H, Kitada C, Taniguchi H, et al. High shear stress can initiate both platelet aggregation and shedding of procoagulant containing microparticles. Blood 1996;88(9):3456-64.

36. Zafrani L, Gerotziafas G, Byrnes C, Hu X, Perez J, Lévi C, et al. Calpastatin controls polymicrobial sepsis by limiting procoagulant microparticle release. Am. J Respir Crit Care Med 2012;185(7):744-55.

37. Johnson Iii BL, Midura EF, Prakash PS, Rice TC, Kunz N, Kalies K, et al. Neutrophil derived microparticles increase mortality and the counter-inflammatory response in a murine model of sepsis. Biochim Biophys Acta Mol Basis Dis 2017; 1863(10):2554-63.

38. Tesse A, Martínez MC, Hugel B, Chalupsky K, Muller CD, Meziani F, et al. Upregulation of proinflammatory proteins through $\mathrm{NF}-\kappa \mathrm{B}$ pathway by shed membrane microparticles results in vascular hyporeactivity. Arterioscler Thromb Vasc Biol 2005;25(12):2522-7.

39. Bardelli C, Amoruso A, Federici Canova D, Fresu L, Balbo P, Neri T, et al. Autocrine activation of human monocyte/macrophages by monocyte-derived microparticles and modulation by PPAR $\gamma$ ligands. Br J Pharmacol 2012;165(3):716-28.

40. Johnson BL, Kuethe JW, Caldwell CC. Neutrophil derived Microvesicles: emerging role of a key mediator to the immune response. Endocr Metab Immune Disord Drug Targets 2014;14(3):210-17.

41. Gasser O, Schifferli JA. Activated polymorphonuclear neutrophils disseminate anti-inflammatory microparticles by ectocytosis. Blood 2004:104(8):2543-8.

42. Pluskota E, Woody NM, Szpak D, Ballantyne CM, Soloviev DA, Simon DI et al. Expression, activation, and function of integrin $\alpha \mathrm{m} \beta 2$ (Mac-1) on neutrophil-derived microparticles. Blood 2008;112(6):2327-35.

43. Mesri M, Altieri DC. Leukocyte microparticles stimulate endothelial cell cytokine release and tissue factor induction in a JNK1 signaling pathway. $J$ Biol Chem 1999;274(33):23111-18.

44. Smyth SS, McEver RP, Weyrich AS, Morrell CN, Hoffman MR, Arepally GM, et al. Platelet functions beyond hemostasis. J Thrombosis Haemostasis 2009;7(11):1759-66.

45. Icli A, Tayyar S, Varol E, Aksoy F, Arslan A, Ersoy I, et al. Mean platelet volume is increased in infective endocarditis and decreases after treatment. Med Princ Pract 2013;22(3):270-3.

46. Cho SY, Jeon YL, Kim W, Kim WS, Lee HJ, Lee WI, et al. Mean platelet volume and mean platelet volume/platelet count ratio in infective endocarditis. Platelets 2014;25(8):559-61.

47. Tok D, Canpolat U, Tok D, Turak O, Isleyen A, Oksuz F, et al. Association of mean platelet volume level with in-hospital major adverse events in infective endocarditis. Wien Klin Wochenschr 2015;127(5-6):197-202.

48. Karagoz E, Tanoglu A, Dogan M. Mean platelet volume: an emerging prognostic factor of infective endocarditis? Platelets 2015;26(4):370. 